\title{
Terminer sa vie dans la violence
}

\section{Christine Hohl Moinat}

Dr méd., Spécialiste en médecine interne générale, membre de la FMH

Récit anonymisé, inspiré d'une expérience médicolégale en médecine de famille et rendu non-identifiable.

Ici, tout le monde se connaît. C'est un petit village. C'est là que vit Bérénice, seule avec ses deux enfants, Paul et Gaspar. Une petite famille dans une grande maison ouverte sur le monde. On y accueille souvent le rire des enfants, la parole libérée des cercles de femmes, les confidences du grand âge au crépuscule de la vie.

Pour Gaspar, l'école, c'est difficile. Doux rêveur curieux, il peine à être là. Toujours ailleurs, toujours en retard, jamais ses affaires. Les notes ne suivent pas. Mais heureusement, il y a Antoine, le vieil instituteur. Il a toujours su y faire avec les enfants, lui. Et malgré la retraite, il continue. Alors pour Gaspar, Antoine, c'est un peu une bouée de sauvetage. La personne qui lui permet de tenir debout dans un système pas fait pour lui. Entre eux, une belle complicité s'est tissée, faite de rires, de larmes, de cartes postales, et de balades.

Autre jour, autre temps, Bérénice est aussi médecin. De garde ce jour-là. C'est exceptionnel, elle devait être en vacances. Son astreinte débute en douceur avec le décès matinal attendu d'une vieille dame à son domicile. La vie la quittait depuis longtemps. Elle constate puis prend le temps qu'il faut avec l'entourage préparé à la séparation. Une affaire qui roule.

\section{Et soudain, c'est là qu'elle l'entend, la sonnerie du téléphone. Un appel qu'elle aurait aimé ne jamais recevoir, ne jamais devoir écouter.}

Et soudain, c'est là qu'elle l'entend, la sonnerie du téléphone. Un appel qu'elle aurait aimé ne jamais recevoir, ne jamais devoir écouter. Celui qui marquera un avant et un après. Dans sa vie de maman, dans sa vie de médecin. Un message qui lui fera vivre une expérience où elle sait que ce qui compte, c'est d'abord ce que l'on sent, ce que l'on est. Et ensuite, bien après seulement, ce que l'on fait. Même les mots parfois, ne servent à rien.

Uniforme bleu au bout du fil. Le choc. Impossible. Suicide par pendaison. Tout se précipite. Elle ne sait pas pourquoi, elle lâche incrédule: «On parle bien du vieil
Antoine, non?» «Ça va aller Docteur?» Non, bien sûr que non! Ça va pas aller du tout! Mais a-t-elle le choix? De toute manière, faut bien que ça aille, elle se dit. «Laissez-moi cinq minutes pour me reprendre et ça ira.» Non mais quelle idiote! Ça n'ira pas, en fait! Pas encore un suicide! Déjà la dernière garde, le mois dernier, c'était pour elle! Par arme à feu. Mais au moins lui, elle ne le connaissait pas. Un promeneur. Tous ces suicides... Rappelez-moi déjà, dans quelle société on vit exactement?

Encore la veille, Gaspar s'était réjoui de relire une carte postale d'Antoine. Mais comment va-t-elle lui dire, à lui? Comment va-t-il pouvoir continuer à aller à l'école? Il l'aimait tellement...

Alors vite, elle amène les enfants chez la voisine. Bien sûr qu'ils sentent le drame. C'est instinctif, ces choses-là. Mais elle ne dira rien. En route, elle fixe son attention. Elle respire et descend dans ses profondeurs, dans son espace protégé, rien qu'à elle. Son ancrage. Un réflexe indispensable à la bonne pratique de son métier, à sa vie de femme et de maman. Quelques secondes de paix avant la tempête.

Arrivée devant la maison, il y a une voiture inconnue, celle banalisée des uniformes. Parcage laborieux. «Bonjour.» "Il est à l'intérieur.» A l'entrée, accueil bienveillant de la même équipe que lors de sa dernière garde pour le promeneur. Décidément! On file au fond de la grange. Sombre. Et il est là. Pendu au bout d'une corde. Bérénice le reconnaît immédiatement. Immobile. Depuis longtemps déjà. Douloureux, tellement douloureux.

Surtout ne pas trop regarder ce visage. Sentir monter la peur, les larmes aussi. Fuir. Vite s'en aller remplir le constat de décès. A la cuisine, elle reconnaît des détails qui n'avaient pas échappé à Gaspar. Elle se risque à lever les yeux, et croise les photos de cet homme au milieu des siens. Voir son sourire à lui. Douleur immense. Indicible. Elle en tremble.

$\mathrm{Au}$ milieu de cette horreur, la femme en uniforme la touche. Un geste doux. Un geste qui relie. Un geste où l'âme affleure. Quelqu'un accueille sa vulnérabilité. Merci. Et puis tout se suivra très vite. Audition. Soutien des proches. Aucun mot ne sonne juste pour dire un tel 
drame. Elle apprend alors en détail les autres tentatives. La souffrance de la famille. Les angoisses. Oui bien sûr que Bérénice y avait pensé parfois, quand Gaspar lui parlait d'Antoine. Mais c'était impossible à croire, pas jusque-là! Et elle imagine maintenant un peu sa douleur à lui. Son incapacité à saisir les mains tendues. Cet immense vide existentiel que l'on comble avec frénésie. L’anesthésie générale et progressive des sens. Le contrôle de soi permanent et la façade comme être-au -monde. Et cet engrenage infernal de la dépersonnalisation jusqu'à l'étape ultime de la dissociation. Toute cette violence si douloureuse au quotidien.

Elle peine encore tellement à y croire aujourd'hui. Elle se rappelle si bien son regard doux, un être sensible.

Enfin, elle repart, le cour lourd. Reprendre cette route et les mêmes virages. Avec un autre regard, à jamais changé. Bérénice s'arrête en chemin au bord de la rivière. Impossibilité de rentrer maintenant. Nécessité de partager cet indicible avec quelqu'un. Elle sait que ses enfants sentiront sa profonde tristesse à elle. Et Gaspar surtout. Il aura besoin d'authenticité, de cohérence. Et elle lui doit bien ça. Mais Bérénice sait aussi qu'elle doit à cette famille de ne rien dire du tout. Pas maintenant. Et pour toujours peut-être. Gaspar, lui, apprendra la nouvelle quoi qu'il arrive. Mais il n'est pas obligé de savoir que sa maman a vu l'horreur.
Alors elle partagera avec un collègue de confiance. Gratitude pour cet espace offert qui lui permet de remettre de l'ordre dans sa tête, dans son cœur. Retrouver le chemin de son âme avant de rentrer chez elle. Ainsi elle peut reprendre pied dans son rôle de maman. Au moins un peu et sans conflit de loyauté. Parce que dès le seuil franchi, tout restera à faire. Etre vraie mais respecter le secret. Vivre avec l'image violente qui reviendra sans cesse. Avec cette absence brutale. Trouver les mots justes pour dire la mort. Dire le suicide. Accompagner les questions sans réponse. Accompagner ceux qui restent. Traverser toute cette tristesse pour continuer de jouer, chanter, danser, rire et construire des cabanes avec Gaspar. Tout un chemin qui ne pourra se faire que lentement, sans sauter une étape. Pour les uns comme pour tous les autres. Parce que le suicide nous touche toutes et tous violemment dans nos intimes profondeurs. Dans notre être-au-monde. De loin et de près.

Pour ce drame, Bérénice trouvera elle aussi la place qui est juste dans son cœur de maman médecin. Et ce qui la sauvera du désespoir une fois encore, c'est son amour, sa quête pour tout ce qui révèle l'âme du monde. La clarté tout comme l'ombre et ses mystères. Elle choisira alors de continuer à aimer. A enchanter. A rêver l'obscur ${ }^{1}$. Parce que la vie renaît. Toujours. Quoi qu'il arrive. Quoi qu'on en pense. Quoi qu'on en dise.

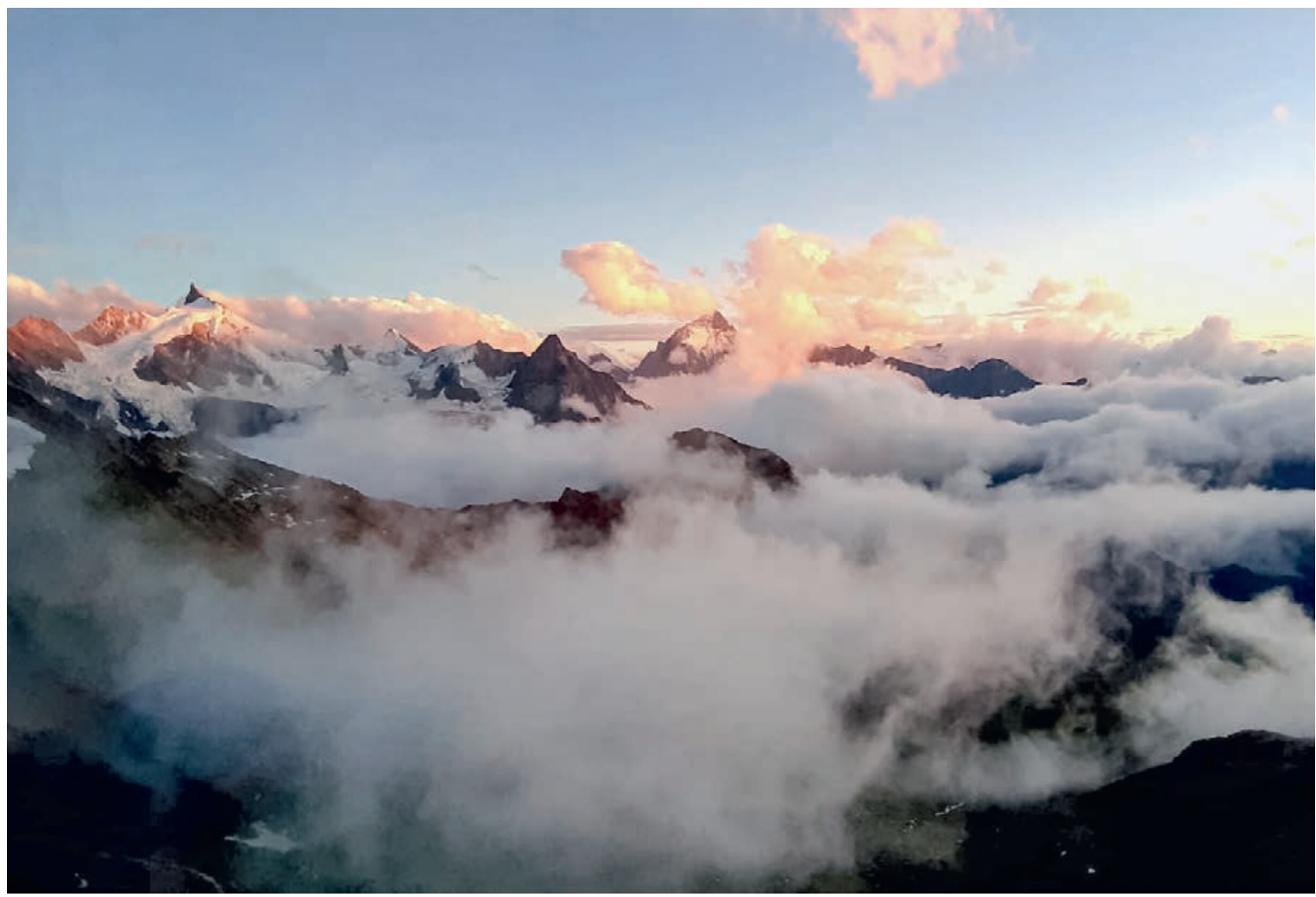

Vue du cercle impérial, août 2019 ( Bertrand Alluin). 